\title{
A note on the WGC, effective field theory and clockwork within string theory
}

\author{
Luis E. Ibáñez ${ }^{a}$ and Miguel Montero ${ }^{b}$ \\ ${ }^{a}$ Departamento de Fúsica Teórica and Instituto de Física Teórica UAM/CSIC, \\ Universidad Autónoma de Madrid, \\ Cantoblanco, 28049 Madrid, Spain \\ ${ }^{b}$ Institute for Theoretical Physics and Center for Extreme Matter and Emergent Phenomena, \\ Utrecht University, \\ Princetonplein 5, 3584 CC Utrecht, The Netherlands \\ E-mail: luis.ibannez@uam.es, m.montero@uu.nl
}

ABSTRACT: It has been recently argued that Higgsing of theories with $\mathrm{U}(1)^{n}$ gauge interactions consistent with the Weak Gravity Conjecture (WGC) may lead to effective field theories parametrically violating WGC constraints. The minimal examples typically involve Higgs scalars with a large charge with respect to a $\mathrm{U}(1)$ (e.g. charges $(Z, 1)$ in $\mathrm{U}(1)^{2}$ with $Z \gg 1)$. This type of Higgs multiplets play also a key role in clockwork $\mathrm{U}(1)$ theories. We study these issues in the context of heterotic string theory and find that, even if there is no new physics at the standard magnetic WGC scale $\Lambda \sim g_{\mathrm{IR}} M_{P}$, the string scale is just slightly above, at a scale $\sim \sqrt{k_{\mathrm{IR}}} \Lambda$. Here $k_{\mathrm{IR}}$ is the level of the IR U(1) worldsheet current. We show that, unlike the standard magnetic cutoff, this bound is insensitive to subsequent Higgsing. One may argue that this constraint gives rise to no bound at the effective field theory level since $k_{\mathrm{IR}}$ is model dependent and in general unknown. However there is an additional constraint to be taken into account, which is that the Higgsing scalars with large charge $Z$ should be part of the string massless spectrum, which becomes an upper bound $k_{\mathrm{IR}} \leq k_{0}^{2}$, where $k_{0}$ is the level of the UV currents. Thus, for fixed $k_{0}, Z$ cannot be made parametrically large. The upper bound on the charges $Z$ leads to limitations on the size and structure of hierarchies in an iterated U(1) clockwork mechanism.

Keywords: Effective Field Theories, Superstrings and Heterotic Strings, Spontaneous Symmetry Breaking

ArXiv EPrint: 1709.02392 


\section{Contents}

1 Higgsing and WGC constraints 1

2 A string theory embedding $\quad 2$

3 Clockwork 5

$\begin{array}{lll}4 & \text { Summary } & 7\end{array}$

\section{Higgsing and WGC constraints}

There has been recently a revived activity in trying to characterize theories which cannot possibly be embedded into a consistent theory of quantum gravity (and hence belong to the swampland [1-3]) from those which can, see [4-26] for a partial list of recent references. The classical constraint is the Weak Gravity Conjecture (WGC) [1-3] which, as applied to a U(1) theory, states that a particle with charge $q$ and mass

$$
m^{2} \leq q^{2} g^{2} M_{p}^{2}
$$

must exist in the low energy spectrum. Equation ((1.1)) only becomes a constraint on the low energy effective field theory if we can somehow restrict $q$ to be not too large. On the other hand, the magnetic version of the constraint indicates there must be an associated scale of new physics at

$$
\Lambda^{2} \simeq g^{2} M_{p}^{2}
$$

This is a genuine constraint on the cutoff of the effective field theory, which unlike (1.1) does not depend on any unknown quantities. So making $g \ll 1$ is not an innocent weakly coupled limit but implies also a new physics scale. The strongest support for the WGC comes from not having found any counterexamples in string theory, although no formal proof exists at the moment. This type of bounds may be generalized from particles to instantons, domain walls and branes leading to interesting constraints on axion cosmology, relaxions and other interesting physical systems [1-26].

An important question is whether one can identify effective field theories which can be ruled out on the basis of the WGC alone. In particular if we find an effective low-energy theory violating eq. (1.1), can we conclude that the theory is in the Swampland? In ref. [27] it was claimed that the answer to this question is no, at least without introducing extra assumptions. In that reference, an explicit example was shown in which, starting from a theory obeying the WGC, Higgsing can lead to an effective theory parametrically violating eq. (1.2). So from a low energy analysis we cannot conclude whether a theory is consistent or not. It is interesting to review this simple model before we go to its string theory version. 
There is a $\mathrm{U}(1)_{1} \times \mathrm{U}(1)_{2}$ theory with gauge couplings $g_{1,2}$ and particles $\psi_{1,2}$ with charges $(1,0)$ and $(0,1)$ respectively. We assume they have masses $m_{1,2}$ consistent with a bound as in eq. (1.1). Consider now a massless scalar with charges $(Z, 1)$ getting a vev $v$. The gauge symmetry is broken like $\mathrm{U}(1)_{1} \times \mathrm{U}(1)_{2} \rightarrow \mathrm{U}(1)$ with a massless gauge linear combination (we take for simplicity $g_{1,2}=g$ and $Z \gg 1$ here)

$$
A=A_{2}-\frac{1}{Z} A_{1}=\left(-\frac{1}{Z}, 1\right) \cdot \vec{A}
$$

The latter equality shows that $A$ has norm 1 , modulo $1 / Z^{2}$ corrections. Now the field $\psi_{1}$ couples to the massless gauge boson with an effective charge $q_{1}=-1 / Z$. One can make in principle $Z$ parametrically large, so that $((1.2))$ would predict the existence of new physics at a scale $\simeq(g / Z) M_{p}$. If there are no fields in the EFT other than $\psi_{1}, \psi_{2}$ and the gauge fields, there is no new physics scale at $\simeq(g / Z) M_{p}$, and the magnetic WGC fails under Higgsing in this particular model.

As discussed in [30], the above reasoning makes the implicit assumption that the UV theory before Higgsing (with fields $\psi_{1}, \psi_{2}, A_{1}, A_{2}$, and nothing else) is not in the Swampland to begin with. Maybe this is not the case, which begs the question; or maybe, whenever we have a sector like the one discussed above, the full theory behaves in such a way that after Higgsing the new EFT cutoff is indeed $(g / Z) M_{P}$. In the latter point of view, advocated in [30], variants of the WGC are valid constraints on EFT's which are not in the Swampland, and the objections raised in [27] and which we have just reviewed do not apply.

Nevertheless, presently we know of no way to exclude examples like the one just discussed, so we will take an agnostic point of view and try to find other similar constraints. We will not be able to do this in general but, as we will see shortly, in a restricted class of string models, we can do better. Namely, we will be able to write an upper bound on the EFT cutoff which is invariant under Higgsing. Thus, in this class of models, we have an alternative to the magnetic WGC which is free of the potential issues discussed above.

\section{$2 \quad$ A string theory embedding}

Let us consider now a string theory $4 \mathrm{D}$ compactification with some $\mathrm{U}(1)^{N}$ gauge group and charged matter fields. We consider here heterotic compactifications, which are particularly versatile in producing massless spectra with a variety of charges, sometimes quite large. The heterotic compactifications we consider can have both non-Abelian $G$ or Abelian groups $\mathrm{U}(1)^{N}$, and the gauge and gravity interactions are unified as (we use conventions as in [28])

$$
\alpha_{G} k_{G}=\alpha_{i} k_{i}=16 \frac{M_{S}^{2}}{M_{p}^{2}}
$$

where $M_{S}^{2}=1 / \alpha^{\prime}, \alpha_{G}, \alpha_{i}$ are the corresponding fine-structure constants and $k_{G}$ is the KacMoody level associated to each non-Abelian gauge factor (a positive integer). This formula is a tree-level result, valid for U(1)'s arising from dimensional reduction of the $10 \mathrm{~d}$ heterotic gauge fields. From the worldsheet perspective, these are precisely the U(1)'s whose vertex 
operators are constructed out of worldsheet currents of the form $\sum_{I} Q_{I} \partial X_{I}$, where the $X_{I}$, $I=1, \ldots, 16$ are the sixteen chiral gauge bosons in the bosonic formulation of the heterotic. As discussed in [28], this formula can be proven directly from dimensional reduction of the 10d supergravity effective action; we thus restrict to heterotic compactifications within the reach of supergravity. It can also be proven directly in worldsheet terms [29] for general non-abelian current algebras; in our context, the result for the $10 \mathrm{~d}$ heterotic current algebra extends to abelian factors which arise after the gauge group is broken by compactification, since at tree-level, the relationship between the $10 \mathrm{~d}$ and $4 \mathrm{~d}$ gauge couplings it is just a volume factor.

The formula also assumes no kinetic mixing in the Abelian sector; this will be generalized later on. In the simplest models $k_{G}=1$ whereas for U(1)'s $k_{i}$ is a normalization factor of each Abelian factor which depends on the choice of $U(1)$ generator. It is a positive rational number (integer if all particle charges are chosen integer) which in principle may be arbitrarily large. To be more precise, with the world-sheet current for a U(1) defined as $J_{Q}=i Q_{I} \partial X_{I}$, with OPE expansion

$$
J_{Q}(z) J_{Q}(w) \simeq \frac{Q^{2}}{(z-w)^{2}}+\ldots
$$

one has $k_{1}=2 Q^{2}$. Here $X_{I}, I=1-16$ are the heterotic gauge left-moving coordinates. Note that although the value of $k_{i}$ is convention dependent, the ratios of the charges of the massless particles $q^{2} / k_{i}$ are convention independent.

Reference [30] proved a sublattice version of the WGC which applies precisely to perturbative heterotic compactifications. For a $\mathrm{U}(1)^{N}$ factor, modular invariance of the worldsheet CFT leads to the existence of states with

$$
q_{i}=l_{i} k_{i}, \quad m^{2} \leq M_{P}^{2} \sum_{i}\left(g_{i}^{2} k_{i}^{2} l_{i}^{2}\right), \quad l_{i} \in \mathbb{Z}
$$

The states have charges in a sublattice with charges $\left(0, \ldots k_{i}, \ldots 0\right)$, and their masses satisfy eq. (1.1).

Consider now a $\mathrm{U}(1)^{N}$ subgroup in some heterotic compactification. Applying blindly eq. (1.2) to this case we would expect $N$ different new physics cut-offs $\Lambda_{i}^{2} \simeq g_{i}^{2} M_{p}^{2}$. As discussed above, these may turn out to yield new physics or not, depending on the particular implementation of Higgsing in the theory - but in any case, it is impossible to know without significant additional stringy input.

However, all these models have a clear upper bound on the EFT cutoff, namely the string scale $\Lambda^{2} \simeq M_{s}^{2}$ - and we can rewrite it in terms of the low energy theory data. In this way we get a new upper bound on the cutoff, independently of the magnetic WGC, which is

$$
\Lambda_{i}^{2}=\alpha_{i} k_{i} M_{p}^{2} .
$$

Unlike the original magnetic WGC, this new cutoff scale is not motivated by any black hole considerations, but by stringy physics. Indeed, it is just the string scale! We will now make some considerations about this new cutoff scale: 
- As it stands now, one cannot write down the cutoff solely from effective field theory data, since the levels $k_{i}$ do not show up anywhere in the effective field theory; they are extra UV information. This does not mean that $((2.4))$ is useless; as we will see below in section 3 , just assuming that the theory has a heterotic stringy completion is enough to constrain the model in some cases.

- Just like the magnetic WGC, equation (2.4) only works in a basis where the charges are integer quantized and there is no kinetic mixing between the different U(1)'s; the case with kinetic mixing will be discussed momentarily.

- The cutoff is invariant even after Higgsing. From the stringy perspective, this has to be the case, since the string scale is clearly insensitive to EFT phenomena such as Higgsing, and the formula $((2.4))$ holds both before and after Higgsing. We will nevertheless see how this happens explicitly, from the EFT point of view.

Our starting point is a $\mathrm{U}(1)^{N}$ theory with charge lattice $\mathbb{Z}^{N}$. In this basis, in which the charge lattice is standard, the U(1)'s are not canonically normalized. The two point functions of the corresponding CFT currents satisfy

$$
\left\langle J_{i}(x) J_{j}(y)\right\rangle \propto \frac{k_{i j}}{(x-y)^{2}},
$$

where $k_{i j}$ is a positive-definite symmetric matrix with integer entries, the level matrix k. Equation (2.5) also tells us the 2-point function of photons in the effective field theory, which allows us to relate it to the gauge coupling matrix $\alpha$ in the kinetic term

$$
\frac{1}{16 \pi} \int \vec{F} \wedge *\left(\alpha^{-1} \vec{F}\right)
$$

directly via a generalization of (2.4),

$$
\mathbf{k}=16 \frac{M_{S}^{2}}{M_{P}^{2}} \alpha^{-1} .
$$

The columns of $\mathbf{k}$ also define a sublattice of $\mathbb{Z}^{N}$. The sublattice WGC says precisely that this sublattice has superextremal states.

We wish to show that (2.7) is invariant under Higgsing. Suppose we have $J$ linearly independent fields, $J<N$, with charge vectors $\vec{v}_{j}, j=1, \ldots J$, which acquire a vev, and let $\mathbf{V}$ be the $J \times N$ matrix which has the $\vec{v}_{j}$ as its rows. Then, Higgsing imposes the condition $\mathbf{V} \vec{A}_{\mathrm{IR}}=0$, so that $\vec{A}_{\mathrm{IR}}$ lives in the kernel of $\mathbf{V}$. Let $\left\{\vec{n}_{i}\right\}$, $i=1, \ldots N-J$ be a orthonormal basis of $\operatorname{ker} \mathbf{V}$, and $\mathbf{N}$ a matrix which has these for rows. Then the charge lattice after Higgsing is the $(N-J)$-dimensional sublattice of vectors of the form

$$
\Lambda_{\mathrm{IR}}:\left\{\vec{v} \in \mathbb{R}^{N-J}: \vec{v}=\mathbf{N} \vec{q}, \quad \vec{q} \in \mathbb{Z}^{N}\right\} .
$$

Naturally, we should also restrict the gauge coupling and the level matrix to the

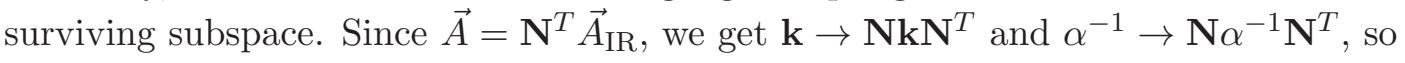
that (2.7) still holds after Higgsing. 
However, we are not done yet because $\mathbf{N k N}^{T}$ is not really a level matrix in the same sense that $\mathbf{k}$ was in the unhiggsed theory. We defined $\mathbf{k}$ to be the charge matrix in a basis where the charge lattice is $\mathbb{Z}^{N-J}$, while $\Lambda_{\mathrm{IR}}$ defined in (2.8) won't be in general. This can be achieved by a further general linear transformation $\mathbf{A}$, which will again preserve (2.7).

In conclusion, it is indeed the case that eqs. (2.4) and (2.7) also apply after Higgsing.

- The cutoff we obtained is secretly none other than the string scale. In any effective field theory coming from a large volume heterotic compactification, the Kaluza-Klein scale $M_{K K}$ is lower than the string scale, so the actual cutoff of the four-dimensional effective field theory will be much lower than (2.4). In any case, (2.4) is always an upper bound for the EFT cutoff.

- Unlike the WGC, we make no claim of (2.4) being a universal constraint which should always apply in a consistent quantum theory of gravity. Rather, it has the same range of validity as the worldsheet proof of the lattice WGC in [30]: it works for closedstring U(1)'s.

Let us consider now the previous $\mathrm{U}(1)^{2}$ toy model embedded into such heterotic setting. Let us take for simplicity $g_{1}=g_{2}=g_{0}$ and $k_{1}=k_{2}=k_{0}$. Consistency with the weakest form of the WGC is still guaranteed, since the particle $\psi_{2}$ with charge 1 under the unbroken $\mathrm{U}(1)$ will obey the first condition (2.4). The same discussion applies now as in the field theory case, and a particle $\psi_{1}$ with charge $(1,0)$ will couple with a strength $\simeq g / Z$ after Higgsing. This sets the quantum of charge for $A_{\mathrm{IR}}$, so that the charge lattice is simply $\mathbf{Z} / Z$; we get to the canonical normalization simply by multiplication by $Z$, which yields

$$
k_{\mathrm{IR}}=Z^{2} k_{0}, \quad \alpha_{\mathrm{IR}}=\frac{\alpha_{0}}{Z^{2}} .
$$

Taking this into consideration, equation (2.4) will predict the existence of a new physics scale at

$$
\Lambda_{s}^{2} \simeq k_{\mathrm{IR}} \alpha_{\mathrm{IR}} M_{p}^{2}=M_{S}^{2}
$$

\section{Clockwork}

As mentioned before, the level of the current algebra $k$ is not part of the information readily available to the effective field theorist, so it is unclear how to even compute the stringy cutoff. We will now input some extra information about the spectrum, which will be enough to show that the model of [27] cannot be consistently embedded in the present setup, at least for parametrically large $Z$.

We will also see that the Clockwork mechanism [34-39], which for our purposes here is essentially $N$ successive Higgsings, is also constrained, since the charges $Z_{i}$ of each step are constrained, and $N$ is constrained as well. One of the typical uses of the Clockwork is the dynamical generation of tiny gauge couplings in a natural way, since we roughly have $g_{\mathrm{IR}} \sim g_{\mathrm{UV}} / Z^{N}$. The bounds on $Z$ and $N$ mean that there is a limit to the hierarchy that can be achieved in this way - although as we will see it is not a too stringent one. 
Let us recall that in string theory massless fields cannot have arbitrarily large charges or live in arbitrarily high dimensional representations. Massless states obey certain BPS-like conditions which strongly constrain their charges. The mass formula for e.g. left-movers in the string spectrum has the form (see e.g. [28])

$$
\frac{\alpha^{\prime} M^{2}}{4}=h_{\mathrm{KM}}+h_{\mathrm{int}}+N_{\mathrm{osc}}-1
$$

where $h_{\text {int }}$ is the contribution to the conformal weight of the state coming from the internal compact space, $h_{\mathrm{KM}}$ is the contribution coming from Kac-Moody gauge degrees of freedom (from the $E_{8} \times E_{8}$ or $\operatorname{Spin}(32)$ lattice), and $N_{\text {osc }}$ counts oscillators. Thus for a particle to be in the massless spectrum a necessary condition is to have $h_{\mathrm{KM}} \leq 1$. On the other hand the gauge conformal weight of a particle transforming in non-Abelian representations $R_{r}$ and transforming under Abelian groups $\mathrm{U}(1)_{s}$ with charge $q_{s}$ and normalization $k_{s}$ is given by [31-33]

$$
h_{\mathrm{KM}}\left(R_{r}, k_{r}, k_{s}\right)=\sum_{r} \frac{C_{r}}{k_{r}+c_{r}}+\sum_{s} \frac{q_{s}^{2}}{k_{s}}
$$

where $k_{r}$ is the KM level of the associated non-Abelian factor, $c_{r}$ is the associated dual Coxeter number (e.g. $c_{r}=N$ for $\mathrm{SU}(N)$ ), and $k_{s}$ is the normalization of the Abelian factors. $C_{r}$ is the quadratic Casimir of the representation $R_{r}$.

Coming back to the $\mathrm{U}(1)^{2}$ toy model, for a scalar in the representation $(Z, 1)$ to be Higgsed (at least by an effective field theory mechanism), it has to be in the string massless spectrum, hence it must have $h_{\mathrm{KM}} \leq 1$. It is clear from the last term in eq. (3.2) that then there is a bound $Z^{2} \leq k_{0}$ and that for fixed $k_{0}$ the value of $Z$ cannot be made parametrically large, one rather has

$$
Z^{2} \leq k_{0}=\frac{16}{\alpha_{0}} \frac{M_{S}^{2}}{M_{p}^{2}} .
$$

So in a consistent embedding of this model in a heterotic string compactification, we would get a UV cutoff

$$
\Lambda^{2} \leq \alpha_{\mathrm{IR}} Z^{2} k_{0} M_{P}^{2} \leq \alpha_{\mathrm{IR}} k_{0}^{2} M_{P}^{2}
$$

Let us recap for a moment and see what we've got so far. As already pointed out in [27], we have obtained a model by Higgsing in which the gauge coupling can be made as tiny as one wants, and in which nothing is happening at the magnetic WGC scale $\Lambda_{\text {mag. }}^{2} \sim \alpha_{\mathrm{IR}} M_{P}^{2}$. Whether a model with these properties can actually embedded in String Theory is not known. But, even if it can, equation (2.4) tells us that the string scale is just a factor of $k_{0}$ times higher, so we do get a light cutoff assuming that $k_{0}$ is not too high. Equivalently, in stringy setups we cannot take $Z$ to be parametrically large, and thus get a tiny IR gauge coupling, unless $k_{0} \gg 1$ to begin with. But (3.3) tells us that this is equivalent to starting with a tiny gauge coupling in the UV anyway, and begs the question. On top of this, there are no explicit stringy examples with parametrically large $k_{0}$. We conclude that the model of [27] can only be consistently embedded in a heterotic compactification - or in general in the closed string sector of any compactification whatsoever - if $Z$ is not too large. 
By iteration of the kind of Higgsing considered in the field theory toy model through $N$ factors in a $\mathrm{U}(1)^{N}$ gauge group, with equal charge $Z$ in all Higgsings, one can obtain light fields with highly suppressed charges of order $Z^{-N}$. This is the clockwork mechanism of refs. [34-39] applied to Abelian gauge interactions. If we were successful in embedding such a model into the heterotic string, the suppression would be bounded as

$$
\frac{1}{Z^{N}} \geq \prod_{i=1}^{N} \frac{1}{k_{i}^{1 / 2}}=\prod_{i=1}^{N}\left(\frac{\alpha_{i}^{1 / 2} M_{p}}{4 M_{S}}\right),
$$

so that there is only supression if $\alpha_{i} \lesssim 16 M_{S}^{2} / M_{p}^{2}$. Finding a specific heterotic $\mathrm{U}(1)^{N}$ model with $N$ sufficiently large and the required Higgs fields would probably be challenging though. The bound (2.4) implies that the string scale is no further away than

$$
\Lambda^{2} \leq \alpha_{\mathrm{IR}} k_{0}^{2 N} M_{P}^{2}
$$

While the mechanism works in principle, we should also note that in the heterotic setting, $N$ is bounded from above as well. The left-moving central charge in heterotic string theory is fixed by conformal invariance to be $c_{L}=22$ (26 from bosonic string theory minus four of the noncompact bosons). On the other hand, a Kac-Moody algebra with group $g$ and level $k$ has a contribution to the central charge

$$
c_{\mathrm{KM}}=\frac{k \operatorname{dim}(g)}{k+c_{r}},
$$

and in particular a $\mathrm{U}(1)^{N}$ factor contributes $N$ to the central charge. It follows that, in any stringy embedding of the clockwork, $N \leq 22 .{ }^{1}$ If the model also includes the SM, the extra contribution to the central charge from the SM gauge fields is

$$
c_{\mathrm{SM}}=\frac{8 k_{\mathrm{SU}(3)}}{k_{\mathrm{SU}(3)}+3}+\frac{3 k_{\mathrm{SU}(2)}}{k_{\mathrm{SU}(2)}+1}+1 \geq 4
$$

which lowers the bound to $N \leq 18$. In fact in generic Calabi-Yau (CY) compactifications of the heterotic string which feature no enhanced $\mathrm{U}(1)$ 's one rather has $N \leq 12$.

\section{Summary}

Summing up, we have seen that it is a priori possible that within string theory Higgsing may produce supressed effective gauge charges (e.g. of order $g / Z$ using $(Z, 1)$ Higgsing). The mild/sublattice version of the WGC is always respected, but there may be no new physics at the IR magnetic scale, as pointed out in [27]. We do not know whether these models can actually embedded in string theory, but we have found that in any case in the heterotic context the string scale $M_{s} \sim \sqrt{k_{\mathrm{IR}}} \Lambda_{W G C}$ is secretly lurking not too far away. Furthermore, this cutoff scale is invariant under further Higgsing. One might try to pull $k_{\mathrm{IR}}=Z^{2} k_{0}$ up, and thus remove the low cutoff, but here is however the additional bound $Z \leq k_{0}$ coming from the Higgs scalar being in the string massless spectrum. Taking this

\footnotetext{
${ }^{1}$ We are not counting graviphotons since these generically have Planck-suppressed couplings.
} 
into account, the string scale is necessarily at or below $k_{0} g_{\mathrm{IR}} M_{P}$. Unlike $k_{\mathrm{IR}}, k_{0}$ is directly related to details of the string compactification, so there is no natural way to make it parametrically large. In fact, we know of no explicit stringy examples with parametrically large $k_{0}$.

The same mechanism also restricts the suppression one can make in an iterative clockwork mechanism. In this clockwork mechanism the suppression cannot be stronger than $k_{0}^{-N}$ where $k_{0}$ is the level of the UV theory. Although $k_{0}$ has to be small, it is still possible to achieve a large hierarchy if $N$ is large enough. So these considerations do not prevent the clockwork from being embedded into heterotic string theory. Of course, finding an actual stringy embedding of the clockwork is a much more complicated issue. In particular, it is not possible to achieve $N \gtrsim 12$ in generic $\mathrm{CY}$ compactifications of the heterotic string.

\section{Acknowledgments}

We thank F. Marchesano, A. Uranga, I. Valenzuela and G. Zoccarato for useful discussions. L. I. also thanks CERN Theory Division where part of this work was carried out. This work has been supported by the ERC Advanced Grant SPLE under contract ERC-2012-ADG20120216-320421, by the grant FPA2012-32828 from the MINECO, and the grant SEV2012-0249 of the "Centro de Excelencia Severo Ochoa" Programme. M.M. is supported by a postdoctoral fellowship from ITF, Utrecht University.

Open Access. This article is distributed under the terms of the Creative Commons Attribution License (CC-BY 4.0), which permits any use, distribution and reproduction in any medium, provided the original author(s) and source are credited.

\section{References}

[1] C. Vafa, The string landscape and the swampland, hep-th/0509212 [INSPIRE].

[2] N. Arkani-Hamed, L. Motl, A. Nicolis and C. Vafa, The string landscape, black holes and gravity as the weakest force, JHEP 06 (2007) 060 [hep-th/0601001] [INSPIRE].

[3] H. Ooguri and C. Vafa, On the Geometry of the String Landscape and the Swampland, Nucl. Phys. B 766 (2007) 21 [hep-th/0605264] [INSPIRE].

[4] T. Rudelius, Constraints on axion inflation from the weak gravity conjecture, JCAP 09 (2015) 020 [arXiv: 1503.00795] [INSPIRE].

[5] M. Montero, A.M. Uranga and I. Valenzuela, Transplanckian axions!?, JHEP 08 (2015) 032 [arXiv: 1503.03886] [INSPIRE].

[6] J. Brown, W. Cottrell, G. Shiu and P. Soler, Fencing in the Swampland: quantum gravity constraints on large field inflation, JHEP 10 (2015) 023 [arXiv: 1503.04783] [INSPIRE].

[7] J. Brown, W. Cottrell, G. Shiu and P. Soler, On axionic field ranges, loopholes and the weak gravity conjecture, JHEP 04 (2016) 017 [arXiv: 1504.00659] [INSPIRE].

[8] B. Heidenreich, M. Reece and T. Rudelius, Weak gravity strongly constrains large-field axion inflation, JHEP 12 (2015) 108 [arXiv: 1506.03447] [INSPIRE]. 
[9] C. Cheung and G.N. Remmen, Naturalness and the Weak Gravity Conjecture, Phys. Rev. Lett. 113 (2014) 051601 [arXiv:1402.2287] [INSPIRE].

[10] A. de la Fuente, P. Saraswat and R. Sundrum, Natural Inflation and Quantum Gravity, Phys. Rev. Lett. 114 (2015) 151303 [arXiv:1412.3457] [INSPIRE].

[11] A. Hebecker, P. Mangat, F. Rompineve and L.T. Witkowski, Winding out of the Swamp: Evading the Weak Gravity Conjecture with F-term Winding Inflation?, Phys. Lett. B 748 (2015) 455 [arXiv: 1503.07912] [inSPIRE].

[12] T.C. Bachlechner, C. Long and L. McAllister, Planckian axions and the weak gravity conjecture, JHEP 01 (2016) 091 [arXiv: 1503. 07853] [INSPIRE].

[13] T. Rudelius, On the possibility of large axion moduli spaces, JCAP 04 (2015) 049 [arXiv: 1409.5793] [INSPIRE].

[14] D. Junghans, Large-field inflation with multiple axions and the weak gravity conjecture, JHEP 02 (2016) 128 [arXiv: 1504.03566] [INSPIRE].

[15] K. Kooner, S. Parameswaran and I. Zavala, Warping the Weak Gravity Conjecture, Phys. Lett. B 759 (2016) 402 [arXiv: 1509.07049] [INSPIRE].

[16] D. Harlow, Wormholes, emergent gauge fields and the weak gravity conjecture, JHEP 01 (2016) 122 [arXiv: 1510.07911] [INSPIRE].

[17] L.E. Ibáñez, M. Montero, A. Uranga and I. Valenzuela, Relaxion monodromy and the weak gravity conjecture, JHEP 04 (2016) 020 [arXiv: 1512.00025] [INSPIRE].

[18] A. Hebecker, F. Rompineve and A. Westphal, Axion monodromy and the weak gravity conjecture, JHEP 04 (2016) 157 [arXiv: 1512.03768] [INSPIRE].

[19] M. Montero, G. Shiu and P. Soler, The weak gravity conjecture in three dimensions, JHEP 10 (2016) 159 [arXiv:1606.08438] [INSPIRE].

[20] H. Ooguri and C. Vafa, Non-supersymmetric AdS and the Swampland, arXiv:1610.01533 [INSPIRE].

[21] B. Freivogel and M. Kleban, Vacua Morghulis, arXiv:1610.04564 [INSPIRE].

[22] D. Klaewer and E. Palti, Super-Planckian spatial field variations and quantum gravity, JHEP 01 (2017) 088 [arXiv: 1610.00010] [INSPIRE].

[23] L. McAllister, P. Schwaller, G. Servant, J. Stout and A. Westphal, Runaway Relaxion Monodromy, arXiv: 1610.05320 [INSPIRE].

[24] A. Herraez and L.E. Ibáñez, An axion-induced SM/MSSM Higgs landscape and the weak gravity conjecture, JHEP 02 (2017) 109 [arXiv:1610.08836] [INSPIRE].

[25] L.E. Ibáñez, V. Martin-Lozano and I. Valenzuela, Constraining neutrino masses, the cosmological constant and BSM physics from the weak gravity conjecture, JHEP 11 (2017) 066 [arXiv:1706.05392] [INSPIRE].

[26] M. Montero, Are tiny gauge couplings out of the Swampland?, JHEP 10 (2017) 208 [arXiv: 1708.02249] [INSPIRE].

[27] P. Saraswat, Weak gravity conjecture and effective field theory, Phys. Rev. D 95 (2017) 025013 [arXiv: 1608.06951] [INSPIRE].

[28] L.E. Ibáñez and A.M. Uranga, String theory and particle physics: An introduction to string phenomenology, Cambridge University Press, Cambridge U.K. (2012). 
[29] P.H. Ginsparg, Gauge and Gravitational Couplings in Four-Dimensional String Theories, Phys. Lett. B 197 (1987) 139 [INSPIRE].

[30] B. Heidenreich, M. Reece and T. Rudelius, Evidence for a sublattice weak gravity conjecture, JHEP 08 (2017) 025 [arXiv: 1606.08437] [INSPIRE].

[31] D.C. Lewellen, Embedding Higher Level Kac-Moody Algebras in Heterotic String Models, Nucl. Phys. B 337 (1990) 61 [INSPIRE].

[32] A. Font, L.E. Ibáñez and F. Quevedo, Higher Level Kac-Moody String Models and Their Phenomenological Implications, Nucl. Phys. B 345 (1990) 389 [InSPIRE].

[33] A.N. Schellekens, Electric Charge Quantization in String Theory, Phys. Lett. B 237 (1990) 363 [INSPIRE].

[34] K. Choi and S.H. Im, Realizing the relaxion from multiple axions and its UV completion with high scale supersymmetry, JHEP 01 (2016) 149 [arXiv:1511.00132] [INSPIRE].

[35] D.E. Kaplan and R. Rattazzi, Large field excursions and approximate discrete symmetries from a clockwork axion, Phys. Rev. D 93 (2016) 085007 [arXiv: 1511.01827] [INSPIRE].

[36] A. Ahmed and B.M. Dillon, Clockwork Goldstone Bosons, Phys. Rev. D 96 (2017) 115031 [arXiv: 1612.04011] [INSPIRE].

[37] G.F. Giudice and M. McCullough, A clockwork theory, JHEP 02 (2017) 036 [arXiv: 1610.07962] [INSPIRE].

[38] N. Craig, I. Garcia Garcia and D. Sutherland, Disassembling the clockwork mechanism, JHEP 10 (2017) 018 [arXiv:1704.07831] [INSPIRE].

[39] H.M. Lee, Gauged U(1) clockwork theory, Phys. Lett. B 778 (2018) 79 [arXiv:1708. 03564] [INSPIRE]. 